\title{
Intravenous to oral antimicrobial stepdown therapy at the Victoria General Hospital, Halifax, Nova Scotia
}

\author{
KR FORWARD MD FRCPC
}

$\mathrm{I}$

N NOVEMBER 1993, THE ANTIBIOTIC UTILIZATION COMMITTEE reached a consensus that implementation of an antibiotic stepdown program at the Victoria General Hospital was highly desirable. In order to gain experience and to

Department of Microbiology and Division of Infectious Diseases, Department of Medicine, Victoria General Hospital, and Dalhousie University, Halifax, Nova Scotia

Correspondence: Dr KR Forward, Department of Microbiology, Victoria General Hospital, 1278 Tower Road. Halifax, Nova Scotia B3H 2Y9, telephone 902-428-3624, Fax 902-428-4432 receive feedback from the medical staff on the strategies we wished to employ, we decided to conduct a pilot study. Ms Poonam Chawla undertook the pilot project as part of her residency training in pharmacy. Drs Kathy Slayter and Walter F Schlech III oversaw the study.

Five units were selected: two general medicine units, cardiology, general surgery and neurology. Patients' medical records were reviewed if they received one or more antibiotics on the hospital's reserve list or if the patient was on two or more antibiotics. Recommenda- 


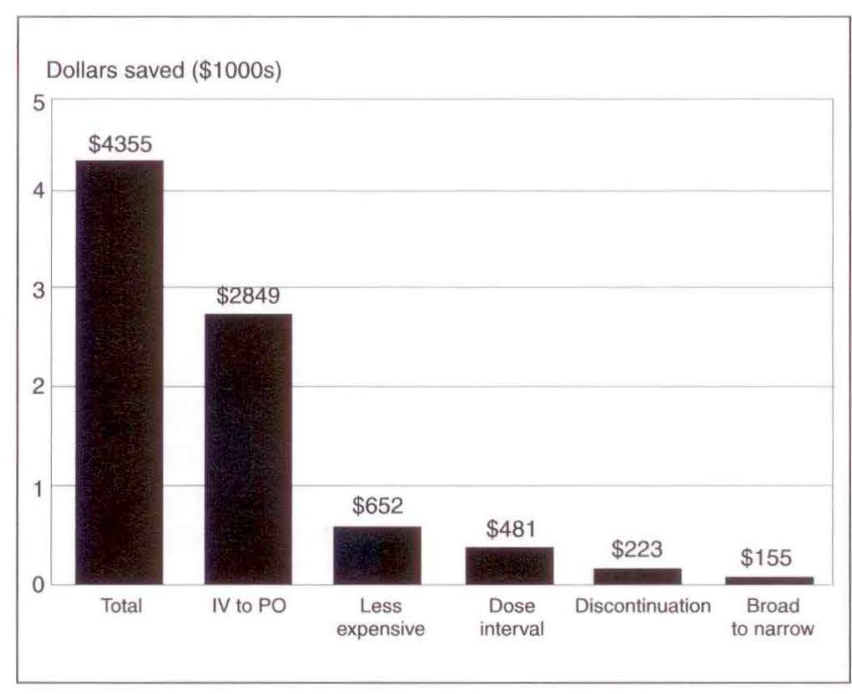

Figure 1) Costs contained versus type of recommendation

tions were placed on the front of the chart but were not incorporated into the permanent medical record. In all, the charts of 86 patients were reviewed and 51 were identified as being eligible for antibiotic streamlining. In all, 89 different recommendations were made; of these, $65(73 \%)$ were accepted by one of the physicians responsible for that patient's care. Sixty $(90.2 \%)$ of the recommendations were either fully followed or the patient was discharged on the recommended oral agent.

Streamlining was defined as being successful if the patient's infection improved and further changes in antimicrobials were not necessary. The recommendations were deemed to have been unsuccessful if the patient's clinical course was judged to warrant an antibiotic change by the responsible physician.

The acquisition costs of the antimicrobials were obtained from the hospital pharmacy. The cost of disposables associated with each intravenous administration of drug was calculated to be $\$ 2.74$. When intravenous to oral recommendations were made, a conservative estimate of two days of savings were calculated. In other cases, the savings for the duration of therapy were calculated. The costs of nursing and pharmacy time, laboratory monitoring of antibiotic levels or toxicity were not calculated, nor were savings related to changes in the length of stay or the consequences of adverse drug reactions.

Table 1 shows the reason why specific recommendations were made. Figure 1 shows the savings associated with different types of recommendations. Stepdown from intravenous to oral therapy was the most frequent recommendation, and was responsible for more than half of the savings. Recommendations in the dosage or the dosing interval were made next most frequently. However, these recommendations realized relatively minor savings.

Other reasons for recommendations included a change from a broad to narrower spectrum antibiotic,
TABLE 1

Reasons for recommendations

- Switch from parenteral to oral route 30

- Change of dose or interval 23

- Change from broad to narrower spectrum 12

- Discontinuation of antibiotics 10

- Change to an equally effective but less 3 expensive antibiotic

- Change from multiple to a single antibiotic 2

- Change to another antibiotic based on 4 antibiotic susceptibility data

TABLE 2

Reasons for noncompliance with recommended changes

- Early discontinuation of antibiotics 7

- Patient died

- New data negated the proposed recommendation

- No response from physician

- Physician judgement that current treatment was more appropriate

recommendations to discontinue antibiotics or to use an equally effective or less expensive antibiotic. In four cases, recommendations were made to change to another antibiotic because susceptibility data suggested a change was appropriate.

Physicians did not always comply with the recommended changes (Table 2). In seven instances, the antibiotic was discontinued early, two patients died, in three cases new information rendered the recommendation invalid, and in nine cases the physician did not respond to the recommendation or judged current therapy to be more appropriate.

Sixty of 65 patients completed therapy or were discharged on the recommended antibiotics. Adverse drug reactions were suspected in two patients. One patient developed a drug fever, and in the second patient trimethoprim-sulphamethoxazole was suspected as being responsible for declining renal function. In one case, the physician altered the dose, disagreeing with the dose which had been recommended. In two patients, active treatment was withdrawn when a palliative approach was adopted.

The level of physician acceptance of our pilot project was very high, and the majority of recommendations were accepted.

In all, $\$ 4,355$ savings were directly attributable to acquisition and administration costs. The decision not to include a control group and the small numbers precluded the accurate collection of information relating to pharmacy, laboratory and nursing costs, and we are unable to determine to what extent length of stay was affected. 
A comprehensive antibiotic streamlining program will probably be formally implemented at the Victoria General Hospital in the late fall of 1994. The pharmacy director is currently negotiating with the hospital administration for the creation of a position dedicated to antibiotic streamlining. Unfortunately, there are relatively few pharmacists with subspecialized training in the field of infectious diseases. We have observed that medical staff are more supportive of a streamlining program if the emphasis is placed on improved patient care, and with the realization that savings can be used in other areas where resources are increasingly scarce. ACKNOWLEDGEMENTS: The author acknowledges the work done by Ms Poonam Chawla and Drs Kathy Slayter and Walter F Schlech III. Their work served as the basis for this report. 


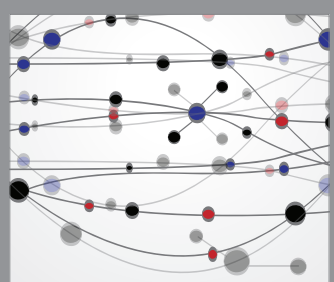

The Scientific World Journal
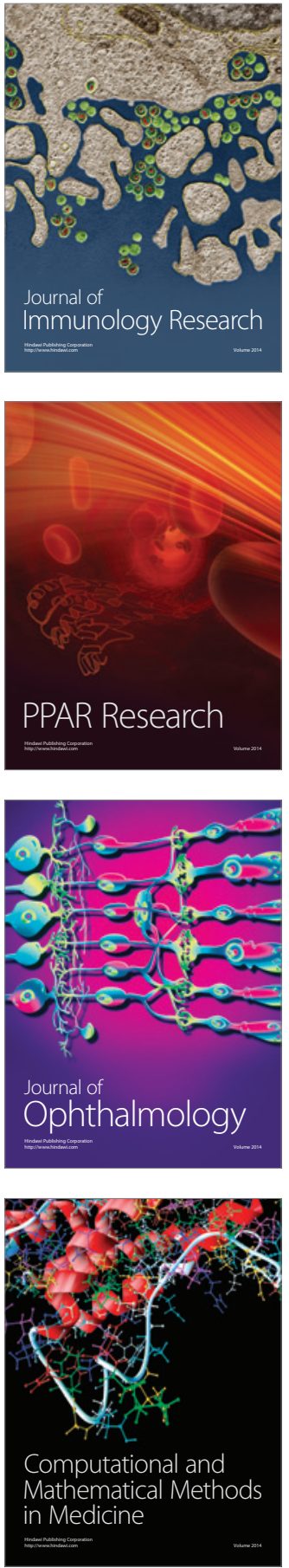

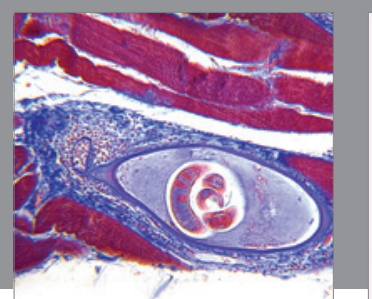

Gastroenterology Research and Practice

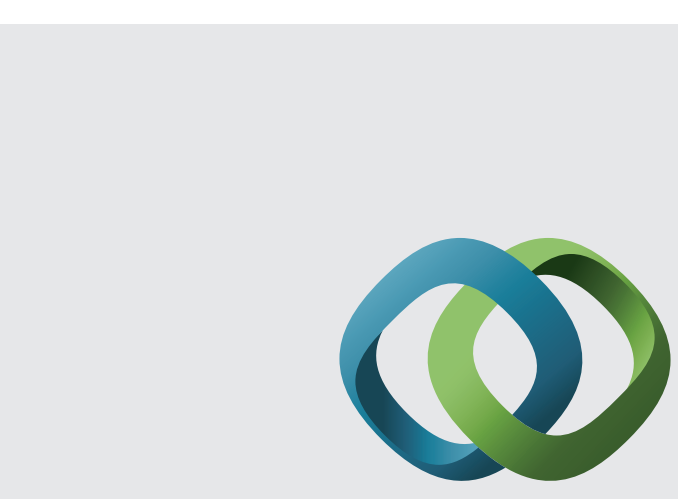

\section{Hindawi}

Submit your manuscripts at

http://www.hindawi.com
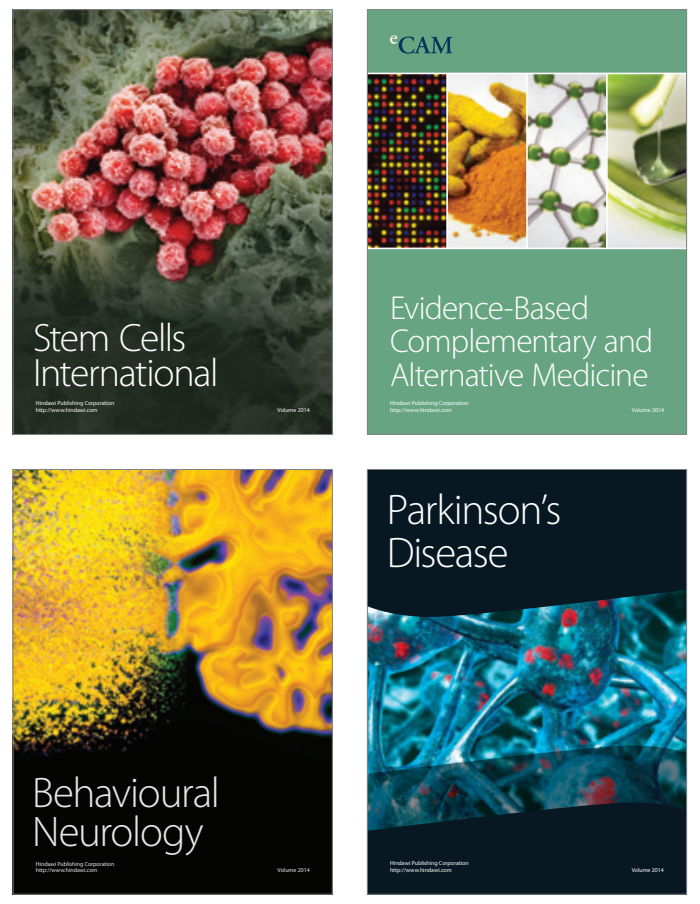
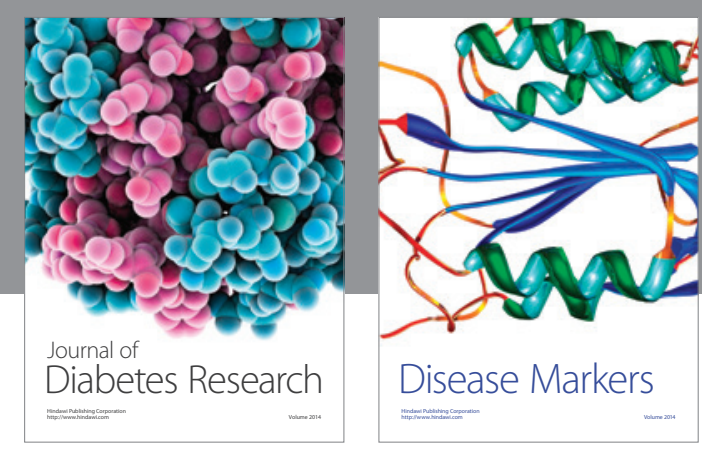

Disease Markers
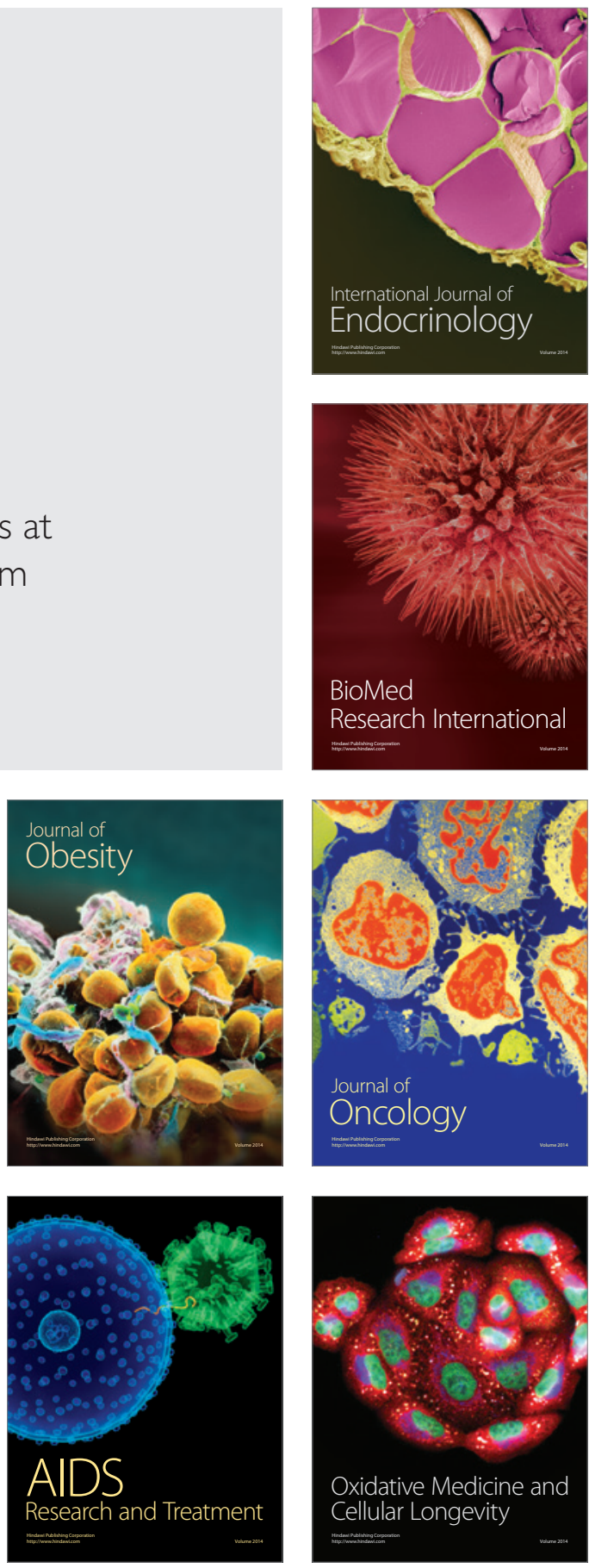